ORIGINAL ARTICLE

\title{
Comparison of Antibiotics and Prebiotics in Treatment of Pelvic Inflammatory Disease
}

\author{
Preeti Amit Kale ${ }^{1}$, Ketan Govindrao Jangale ${ }^{2}$, Amruta Kakade $^{3}$
}

\begin{abstract}
Introduction: Pelvic inflammatory disease (PID) refers to an infectious and inflammatory disorder of the female upper genital tract. It is common among young sexually active individuals and is a major health problem both in developed and developing countries. It is usually a polymicrobial infection; however, Chlamydia trachomatis is the commonest causative agent transmitted sexually. Pelvic inflammatory disease is diagnosed by history and clinical examination. The treatment is initially empiric. Only antibiotics are good in treatment for PID and "antibiotics with prebiotics" are a new promising treatment mode for the same. This comparative study aimed to measure efficiency and compliance of only antibiotics vs antibiotics with prebiotics in the treatment of mild, uncomplicated acute PID.
\end{abstract}

Setting: Multispecialty Hospital Pune. Duration: 6 months.

Materials and methods: A descriptive cross-sectional study was conducted in randomly selected 28 consecutive outpatients with mild uncomplicated acute PID. Comparative outcome of antibiotics and antibiotics with prebiotics in correlation with the reduction of amount, consistency, and malodor of vaginal discharge along with the reduction in fornical tenderness, compliance, and frequency of adverse drug reactions was done.

Results: Among 28 cases, 14 (50\%) were treated with oral antibiotics and 14 (50\%) with oral antibiotics with prebiotics. Comparative analysis on the reduction of amount, consistency, malodor of vaginal discharge, and reduction of fornical tenderness was significant, showing antibiotics with prebiotics more effective than antibiotics. Besides, compliance was better and there were fewer adverse drug reactions with antibiotics with prebiotics.

Conclusion: Antibiotics with prebiotics have better efficiency and compliance over only antibiotics in the treatment of mild, uncomplicated, acute PID.

Keywords: Antibiotics with prebiotics, Chlamydia trachomatis, Pelvic inflammatory disease.

Journal of South Asian Federation of Menopause Societies (2020): 10.5005/jp-journals-10032-1204

\section{INTRODUCTION}

Pelvic inflammatory disease (PID) refers to an infectious and inflammatory disorder of the female upper genital tract, which comprises the uterus, fallopian tubes, adjacent parametrium, and the overlying peritoneum. ${ }^{1}$ Dissemination of infection and inflammation may occur to the abdomen and perihepatic structures. ${ }^{2}$ During the last decade, there is an increasing incidence of PID among sexually active young couples especially commercial sex workers, and has become a major health problem both in the developed and in developing countries. ${ }^{3}$

The exact incidence and prevalence of PID are still not known in Nepal and such a comparative study has not been done yet. WHO in 2005 estimated around 448 million curable new cases of sexually transmitted infections (STIs) in the age group 15-49 years occur annually. ${ }^{4}$ Women of sub-Saharan Africa and Southeast Asia, who are in resource-limited regions, are at increased risk of sequelae and complications. In developed countries, the annual incidence of PID is around 10-20 per 1,000 reproductive age group women. ${ }^{5}$

The PID starts with the infection of the vagina and cervix that ascends. Commonest sexually transmitted causative agent associated with PID is Chlamydia trachomatis (serovars D-K). Other organisms include Neisseria gonorrhoeae, Gardnerella vaginalis, Haemophilus influenzae, and Anaerobes, such as, Peptococcus and Bacteroides species. ${ }^{6}$ Pelvic inflammatory disease is primarily

\begin{abstract}
${ }^{1-3}$ Department of Gynaecology and Obstetrics, AiMS Hospital and Research Center, Pune, Maharashtra, India

Corresponding Author: Preeti Amit Kale, Department of Gynaecology and Obstetrics, AiMS Hospital and Research Center, Pune, Maharashtra, India, Phone: +91 9423585026, e-mail: gynaecward@aimspune.com

How to cite this article: Kale PA, Jangale KG, Kakade A. Comparison of Antibiotics and Prebiotics in Treatment of Pelvic Inflammatory Disease. J South Asian Feder Menopause Soc 2020;8(1):24-26.

Source of support: Nil

Conflict of interest: None

a polymicrobial infection in almost $30-40 \%$ of cases which usually starts with an isolated infection with C. trachomatis or N. gonorrhoeae. ${ }^{7}$ Pelvic inflammatory disease may occur from granulomatous salpingitis caused by Mycobacterium tuberculosis or Schistosoma species in some regions, and it may be commonly associated with HIV infection. ${ }^{8,9}$

Multiple sexual contacts, a prior history of STIs, and sexual abuse are some of the risk factors for PID. ${ }^{10}$ Surgical interventions, such as, curettage, endometrial biopsy, and hysteroscopy breach the barrier of the cervix and thereby predispose ascending infections. ${ }^{11,12}$ Besides, broad-spectrum and frequent use of antibiotics, diabetes mellitus and long-term steroid treatment may predispose to PID. Infertility, ectopic pregnancy, and tubo-ovarian abscess are some of the common complications of $\mathrm{PID}^{13,14}$
\end{abstract}

\footnotetext{
(c) The Author(s). 2020 Open Access This article is distributed under the terms of the Creative Commons Attribution 4.0 International License (https:// creativecommons.org/licenses/by-nc/4.0/), which permits unrestricted use, distribution, and non-commercial reproduction in any medium, provided you give appropriate credit to the original author(s) and the source, provide a link to the Creative Commons license, and indicate if changes were made. The Creative Commons Public Domain Dedication waiver (http://creativecommons.org/publicdomain/zero/1.0/) applies to the data made available in this article, unless otherwise stated.
} 
Acute PID is diagnosed by history and clinical examination. Young woman with multiple sexual contacts, not using any contraception, and residing in STIs prevalent area is a classical highrisk patient for PID. Around $75 \%$ of patients present with abnormal vaginal discharge and around $40 \%$ present with unexpected vaginal bleeding, often post-sexual intercourse. Some common physical findings are tenderness of the uterus, adnexa, and cervical motion. There is no single conclusive test for PID; however, various imaging, laboratory analysis, and procedures can be performed to have the definitive diagnosis. ${ }^{15-17}$

In any suspected case of PID, it is recommended to use broadspectrum antibiotics empirically. Chosen antibiotics need to be effective against $C$. trachomatis, $N$. gonorrhoeae, gram-negative facultative organisms, anaerobes, and streptococci. Treatment also depends on the clinical presentation, complications or sequelae, and culture growth whenever possible. Both oral and parenteral formulations are available for acute symptoms and microbiological cure. Antibiotics are the time tested oral therapy and antibiotics with prebiotics are also available in recent days. After 72 hours of therapy, all patients need to be reassessed to see clinical progress and compliance. Several studies have shown poor compliance with only antibiotics therapy and around $20-25 \%$ of patients with PID remain unrecorded. Centers for Disease Control and Prevention (CDC) recommends antibiotics or antibiotics with prebiotics as a first-line drug for the treatment of Chlamydia infection. Medical treatment with these agents is $95 \%$ effective. ${ }^{18}$ In this comparative study, the treatment regimen considered is either only antibiotics or antibiotics with prebiotics.

The hypothesis of this study is antibiotics with prebiotics better over only antibiotics in the treatment of mild, uncomplicated acute PID and the aim is to test the hypothesis and to measure the outcome in terms of efficacy and compliance.

\section{Materials and Methods}

This study was conducted in Multispecialty Hospital, Pune Maharashtra. A descriptive cross-sectional study in 14 consecutive patients in tertiary care hospital with a working hypothesis of antibiotics with prebiotics is efficacious than only antibiotics in the treatment of acute uncomplicated PID.

\section{Inclusion Criteria}

Women of reproductive age group (15-49 years) attending the gynecology outpatient department having lower abdominal pain and abnormal per vaginal discharge with abdominal and/or pelvic organ tenderness on examination were included for the study.

\section{Exclusion Criteria}

Anyone having pregnancy, uterovaginal prolapse, the recent history of any antibiotic use or any known allergy to study medication, temperature $380^{\circ} \mathrm{C} / 100.40^{\circ} \mathrm{F}$ or higher, rebound tenderness, no clinical improvement after 72 hours of treatment were excluded.

Detailed general and specific history of the patient were taken before subjected to clinical examination.

A pregnancy test with a "test kit" was done for all enrolled patients and they were asked to micturate before the clinical examination. For gynecological examination, women were kept relaxed in dorsal position with the knees flexed. Perineum inspected for any rashes, excoriation, tears, or any signs of inflammation. Per speculum (Cusco's) examination performed in a good source of light. Any abnormality in the vagina and cervix along with the amount, color, consistency, and odor of vaginal discharge were also noted. Per vaginal and bimanual examination was done for the assessment of uterine size, position, mobility, and adnexal condition. Any suspected mass or tenderness felt in the fornices and pouch of Douglas were noted. Pain measured as per numeric pain rating scale who could quantify their pain in given numbers (0-10 for no pain, moderate pain to worst possible pain) and verbal pain intensity scale for those who could express but could not quantify in numbers (no pain, mild, moderate, severe, very severe, and worst possible pain). Patients were randomly selected for treatment with either antibiotics with prebiotics or antibiotics and individually instructed to take the medication group I Tab. Doxy $100 \mathrm{mg} \mathrm{BD}$, Tab. Metro 400 mg TDS; group II Tab. Doxy 100 mg BD, Tab. Metro 400 mg TDS, Tab. Combinorm OD.

Both treatment group patients were followed after 72 hours and then after 14 days. As an outcome measure they were asked about any reduction of vaginal discharge (amount, consistency, and malodor), reduction of fornical tenderness, compliance, and adverse drug reactions of the given medication (Tables 1 to 4 ).

\section{Results}

Out of 28 cases, 14 (50\%) were treated with antibiotics and 14 (50\%) with antibiotics with prebiotics. Comparative analysis on the reduction of amount of vaginal discharge, consistency of vaginal discharge, malodor of vaginal discharge, and fornical tenderness was significant, showing antibiotics with prebiotics to be more effective than only antibiotics (Table 5).

Table 1: Agewise distribution

\begin{tabular}{lll}
\hline Age & No. of patient & Percentage \\
\hline $19-25$ & 05 & 17.85 \\
$26-30$ & 10 & 35.71 \\
$31-35$ & 10 & 35.71 \\
$36-40$ & 03 & 10.71 \\
\hline
\end{tabular}

Table 2: Paritywise distribution

\begin{tabular}{lll}
\hline Parity & No. of patient & Percentage \\
\hline Nullipara & 06 & 21.42 \\
Primipara & 05 & 17.85 \\
Multipara & 17 & 60.71 \\
\hline
\end{tabular}

Table 3: Diabetes mellitus (DM) and non-DM patients

\begin{tabular}{lll}
\hline Partner & No. of patient & Percentage \\
\hline DM & 06 & 21.42 \\
Non-DM & 22 & 78.57 \\
\hline
\end{tabular}

Table 4: Associated medical conditions

\begin{tabular}{lll}
\hline $\begin{array}{l}\text { Associated medical } \\
\text { conditions }\end{array}$ & No. of patient & Percentage \\
\hline DM & 06 & 21.42 \\
Anemia & 02 & 7.1 \\
UTI & 05 & 17.85 \\
Thyroid & 07 & 25 \\
Koch's history & 01 & 3.57 \\
\hline
\end{tabular}


Table 5: Medication

\begin{tabular}{lllll}
\hline & \multicolumn{2}{c}{ Symptoms } & \multicolumn{2}{c}{ Structural } \\
\cline { 2 - 5 } Medication & Relief & No relief & Relief & No relief \\
\hline Only antibiotics & 04 & 10 & 04 & 10 \\
$\begin{array}{l}\text { Antibiotics with } \\
\text { prebiotic }\end{array}$ & 13 & 01 & 13 & 01 \\
\hline
\end{tabular}

\section{Discussion}

In this study, reduction of amount, consistency, and malodor of vaginal discharge along with the reduction of fornical tenderness was significant for antibiotics with prebiotics. Comparing antibiotics and antibiotics with prebiotics, this study showed better compliance and less adverse drug reactions for antibiotics with prebiotics over antibiotics.

Our study has some limitations. First, a small sample size that may only be the tip of the iceberg makes it difficult to draw any conclusion. Second, women of the reproductive age group only were taken into consideration; hence, it is difficult to generalize the result. Third, pain scales being subjective can have an individual bias.

\section{Conclusion}

Antibiotics with prebiotics have better compliance and efficiency than antibiotics only for acute uncomplicated PID. Hence, it can be concluded that antibiotics with prebiotics are a better option in the treatment of a mild, uncomplicated form of acute PID.

\section{References}

1. Westrom L, Joesoef R, Reynolds G, et al. Pelvic inflammatory disease and fertility. Sex Transm Dis 1992;19(4):185-192. DOI: 10.1097/00007435-199207000-00001.

2. Wiesenfeld HC, Hiller SL, Meyn LA, et al. Subclinical pelvic inflammatory disease and infertility. Obstet Gynecol 2012;120(1):3743. DOI: $10.1097 /$ AOG.0b013e31825a6bc9.

3. Fox KK, Behets FM. Vaginal discharge: how to pin point cause? Postgrad Med 1995;98(3):87-90. DOI: 10.1080/00325481.1995.11946044.

4. Low N, Broutet N, Adu-Sarkodie Y, et al. Global control of sexually transmitted infections. Lancet 2006;368(9551):2001-2016. DOI: 10.1016/S0140-6736(06)69482-8.
5. Kamwendo F, Forslin L, Bodin L, et al. Programs to reduce pelvic inflammatory disease-the swedish experience. Lancet 1998;351(Suppl 3):25-28. DOI: 10.1016/S0140-6736(98)90008-3.

6. Mylonas I. Female genital chlamydia trachomatis infection: where are we heading? Arch Gynecol Obstet 2012;285(5):1271-1285. DOI: 10.1007/s00404-012-2240-7.

7. Herzog $\mathrm{SA}$, Althaus $\mathrm{CL}$, Heijne JC, et al. Timing of progression from chlamydia trachomatis infection to pelvic inflammatory disease: a mathematical modeling study. BMC Infect Dis 2012;12:187. DOI: 10.1186/1471-2334-12-187.

8. Sorvillo F, Smith L, Kerndt P, et al. PID, HIV and African-Americans. Emerg Infect Dis 2001;7:927-932. DOI: 10.3201/eid0706.010603.

9. Crossby R, Diclemente RJ, Wingwood GM, et al. Predictors of infection with PID: prospective study of low income African-American adolescent females. Sex Trans Dis 2002;78:360-364. DOI: 10.1136/ sti.78.5.360.

10. Acharya V. Sexually transmitted diseases in gynecological practice. J Obstet Gynecol India 1998;38:3-9.

11. Griger AM, Foxman B. Risk factors in PID: a case control study among college students. Epidemiology 1996;7:182-187. DOI: 10.1097/00001648-199603000-00013.

12. Horowitz B, Eldestein SW, Lippman L. Sexual transmission of PID. Obstet Gynecol 1987;69:883-886.

13. Banikarim C, Chacko MR. Pelvic inflammatory disease in adolescents. Adolesc Med Clin 2004;15(2):273-285. DOI: 10.1016/j. admecli.2004.02.005.

14. Zeger W, Holt K. Gynecologic infections. Emerg med Clin North Am 2003;21(3):631-648. DOI: 10.1016/S0733-8627(03)00039-7.

15. Tukeva TA, Aronen HJ, Karjalainen PT, et al. MR imaging in pelvic inflammatory disease: comparison with laparoscopy and US. Radiology 1999;210(1):209-216. DOI: 10.1148/radiology.210.1.r9 9ja04209.

16. Burnett AM, Anderson CP, Zwank MD. Laboratory confirmed gonorrhea and/or chlamydia rates in clinically diagnosed pelvic inflammatory disease and cervicitis. Am J Emerg Med 2012;30(7):11141117. DOI: 10.1016/j.ajem.2011.07.014.

17. Schoeman SA, Stewart CM, Booth RA, et al. Assessment of best single sample for finding chlamydia in women with and without symptoms: A diagnostic test study. BMJ 2012;345:e8013. DOI: 10.1136/bmj.e8013.

18. Ness RB, Soper DE, Holley RL, et al. Pelvic inflammatory disease evaluation and clinical health $(\mathrm{PEACH})$ study investigators. Effectiveness of inpatient and outpatient treatment strategies for women with pelvic inflammatory disease: a randomized trial. Am J Obstet Gynecol 2001;186(3):929-937. DOI: 10.1067/mob.2002.121625. 